\title{
Wavelength-tunable colloidal quantum dot laser on ultra-thin flexible glass
}

\author{
C. Foucher, B. Guilhabert, N. Laurand and M.D. Dawson \\ Institute of Photonics, SUPA, University of Strathclyde, Glasgow, UK
}

A mechanically-flexible and wavelength-tunable laser with an ultra-thin glass membrane as substrate is demonstrated. The optically-pumped hybrid device has a distributed feedback cavity that combines a colloidal quantum dot gain film with a grating-patterned polymeric underlayer, all on a $30-\mu \mathrm{m}$ thick glass sheet. The total thickness of the structure is only $75 \mu \mathrm{m}$. The hybrid laser has an average threshold fluence of $450 \pm 80 \mu \mathrm{J} / \mathrm{cm}^{2}$ (for 5 -ns excitation pulses) at an emitting wavelength of $607 \mathrm{~nm}$. Mechanically bending the thin-glass substrate enables continuous tuning of the laser emission wavelength over an 18-nm range, from $600 \mathrm{~nm}$ to $618 \mathrm{~nm}$. The correlation between the wavelength tunability and the mechanical properties of the thin laser structure is verified theoretically and experimentally.

Colloidal quantum dots (CQDs) are attractive nanomaterials for the fabrication of photonic devices. They have high photoluminescence efficiency, are compatible with solution-processing techniques and their emission wavelength can be tuned over a wide range by changing their size and/or composition. Consequently CQDs are being developed for devices and applications that include displays, light emitting diodes (LEDs), lasers, bio-imaging and bio-sensing ${ }^{1-5}$. Furthermore, because CQDs can be deposited onto a variety of materials, they also have a role to play in devices for flexible photonics $^{6-8}$. Usually, thin-films of light-emitting materials, e.g. CQDs, are combined with entirely polymeric substrates and structures to enable such devices. Polymers are used because of their high mechanical flexibility ${ }^{9}$. In this paper, we introduce a format of flexible laser based on CQDs using ultra-thin glass. The mechanical properties of the overall structure are such that the laser can be continuously tuned in wavelength by bending the glass membrane.

Glass is the material of choice for many technological solutions, often providing the interface between the 'outside world' and an underlying electronic/photonic core device. It offers advantageous characteristics compared to polymers such as high thermal stability, high chemical resistance, excellent barrier properties and high transmission of visible light ${ }^{10}$. At the same time, mechanical-flexibility in glass sheets can still be obtained if they are made thin enough $(<100 \mu \mathrm{m})^{11}$. Such thin-glass sheets with high optical quality are being developed for the display market, which in turn is opening up opportunities for other photonic devices. While a limited number of optical 
structures made on $75-\mu \mathrm{m}$ or $100-\mu \mathrm{m}$ thick glass substrates have been demonstrated recently (e.g. AM-LCDs, ChLC e-paper and electrophoretic displays ${ }^{12-14}$ ), our report focuses on the demonstration of a laser on flexible glass. CQDs are used to form the gain region of our lasers but we note that the approach is compatible with other types of laser materials as well, e.g. organic semiconductors. We foresee that the laser technology reported here will provide opportunities for, amongst others, biosensing and/or mechanical sensing applications.

In the following, we start by explaining the design and fabrication of the CQD flexible lasers on glass. Then, we describe the optical and mechanical set-ups that were used for the characterisation of the devices. In the last section, we present and discuss the experimental results, including spectra, power transfer functions and mechanical-tunability characteristics of the lasers. In particular, we show that the magnitude of the wavelength shift upon bending results from the interplay between the radius of curvature of the glass substrate and the overall mechanical properties of the 'layered' laser structure.

The schematic in Figure 1 (a) represents the structure of the CQD laser with the thin glass membrane acting as substrate. It is composed of a CQD gain layer ( 160-nm-thick) deposited on top of a onedimensional grating, which is imprinted into the surface of a $43-\mu m$-thick polymer film. The periodicity of the grating, $\Lambda$, is chosen to yield a second-order distributed feedback (DFB) cavity. Vertical out-coupling of the laser is provided through the first order of diffraction while optical feedback within the CQD film is obtained via the second order of diffraction. For this to be the case, $\wedge$ needs to fulfil:

$$
\Lambda=\frac{\lambda}{\mathrm{n}_{\mathrm{eff}}}
$$

In Eq. (1), $\lambda$ approximates the emission wavelength and $n_{\text {eff }}$ is the effective refractive index of the laser mode. For our CQD laser in this work, $\Lambda=390 \mathrm{~nm}$. The resulting DFB resonator sits on top of a 30- $\mu$ m-thick glass membrane. The gain layer is a solid film of CQDs in poly(methyl methacrylate) $(\mathrm{PMMA}){ }^{15}$. Pictures of the fabricated flexible device are shown under white and ultraviolet light illumination, in Figures 1 (b) and 1 (c) respectively.

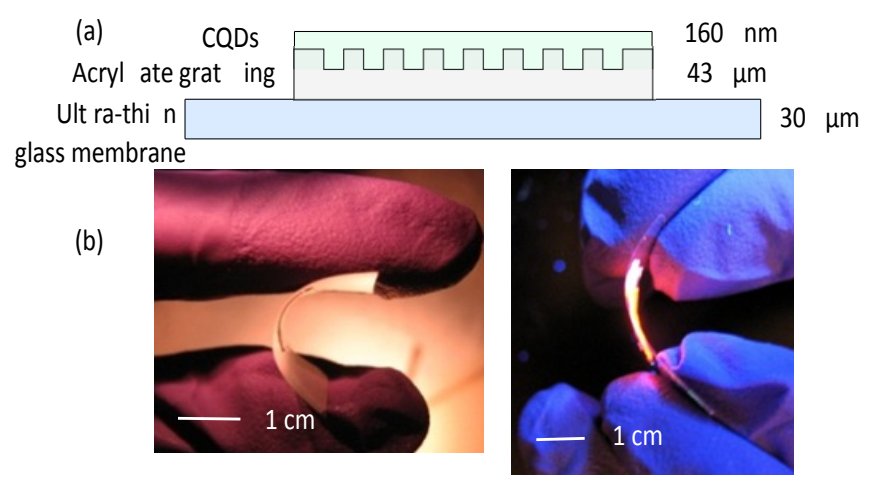

Figure 1: (a) Schematic structure of the laser on a flexible glass substrate. Optical pictures of the mechanically flexible device (b) under white and (c) UV light illumination.

A summary of the full fabrication process of the CQD DFB lasers is detailed in the supplement material.

The DFB lasers are optically pumped by a Q-switched, frequency-tripled Nd: YAG laser emitting 5-ns pulses at a wavelength of $355 \mathrm{~nm}$ and at a $10-\mathrm{Hz}$ repetition rate. The samples are placed at an angle 
of $45^{\circ}$ with respect to the pump beam axis in order to detect the vertical laser emission. ${ }^{16}$ This laser emission was coupled into a 50- $\mu \mathrm{m}$-core optical fibre, itself connected to a CCD-spectrometer with a spectral resolution of $0.13 \mathrm{~nm}$. The full width at half maximum (FWHM) pump spot size on the sample was $57.0 \pm 0.8 \mu \mathrm{m} \times 35.0 \pm 0.6 \mu \mathrm{m}$ as verified by knife-edge measurement. The power transfer function and spectra of the laser were measured. The average threshold quoted in the experimental results section is obtained by averaging several measurements taken at different closely spaced positions (within an area of $2 \times 2 \mathrm{~mm}^{2}$ ) on the surface of the device.

A slightly different set-up was used to study the effect of mechanical bending on the emission wavelength. Figure 2 (a) shows a schematic of this set up. The edges of the laser glass substrate, on both side of the device, were taped onto a mechanical mount (Fig. 2 (a)). Initially, $1.9 \mathrm{~cm}$ separated both sections of the mount. One section could be translated using a micrometre screw while the other section is fixed. To bend the laser (Fig. 2(b)), the distance between the two sections was reduced. We will see later that the laser wavelength shifts as the radius of curvature is reduced or increased. The laser was pumped perpendicularly to the laser surface and the emission is collected on the other side, as shown in Fig. 2 (a), by the same optical fibre/spectrometer system as before. The radius of curvature of the laser could be changed positively or negatively according to the way the substrate was bent, as shown in figure 2 (b), by positioning the sample facing up or facing down on the mount. A positive curvature increases the grating period and engenders a red shift of the emitted wavelength while a negative curvature induces a blue shift (see following section).

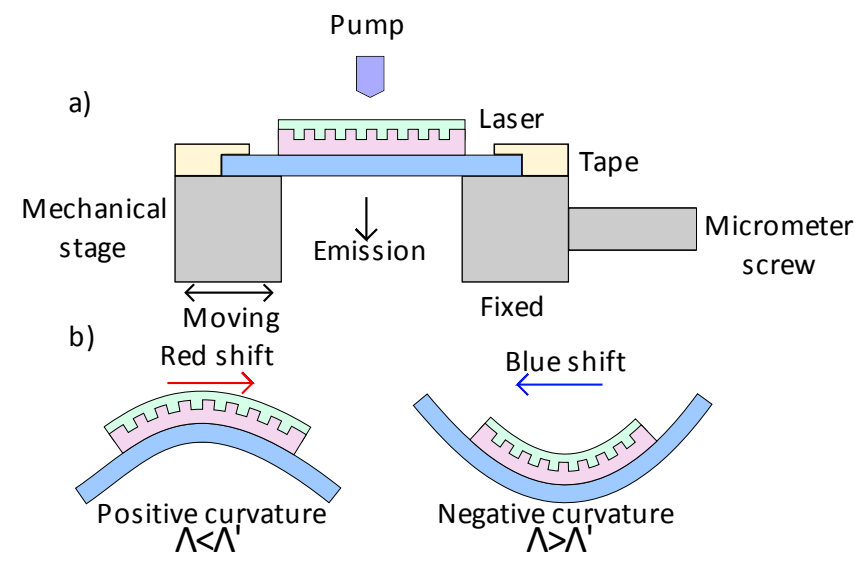

Figure 2: (a) Schematics of the set-up for testing the DFB laser on glass under mechanical bending. (b) Shows the two ways the laser can be bent, increasing or reducing the grating period for, respectively, red shifting or blue shifting the laser emission wavelength.

A typical spectrum and the power transfer function for a 'flat' DFB laser are plotted in Figures 3 (a) and (b), respectively. The oscillation threshold is $375 \mu \mathrm{J} / \mathrm{cm}^{2}$. The spectrum of the CQD laser 2-times above threshold shows a single peak emission wavelength centred at $607.35 \mathrm{~nm}$ and with a FWHM of $0.6 \mathrm{~nm}$, determined by a Gaussian fit of the spectrum. The inset of figure 3 (b) shows more details about the spectral evolution of the laser emission close to the threshold transition. The FWHM of the laser linewidth was between $0.3 \mathrm{~nm}$ and $0.6 \mathrm{~nm}$ across the whole range of operation studied. Two other representative transfer functions measured at different positions on the same sample are plotted in the supplemental material, figure S2. The overall average threshold for this laser is $450 \pm 80 \mu \mathrm{J} / \mathrm{cm}^{2}$. The variation in threshold with position is attributed to possible defects and scattering centres within the film or on the grating surface at particular spatial positions and is not due to a change of the CQD film thickness, as the wavelength is constant within $1.1 \mathrm{~nm}$ over the whole surface of the laser. Other laser devices were fabricated and measured as well and showed similar characteristics with average threshold below $450 \mu \mathrm{J} / \mathrm{cm}^{2}$. This state-of-the-art threshold performance shows that a concentration of $50 \mathrm{mg} / \mathrm{mL}$ is sufficient to obtain laser oscillation with our 
approach whereas the literature often stresses the need for highly concentrated CQD solution (100 to $200 \mathrm{mg} / \mathrm{mL}$ ) to obtain a dense, i.e. close packed, film after spin-coating. ${ }^{17}$ This low threshold is influenced by the thickness of the film obtained by combining an appropriate concentration and spin-coating speed.

(a)

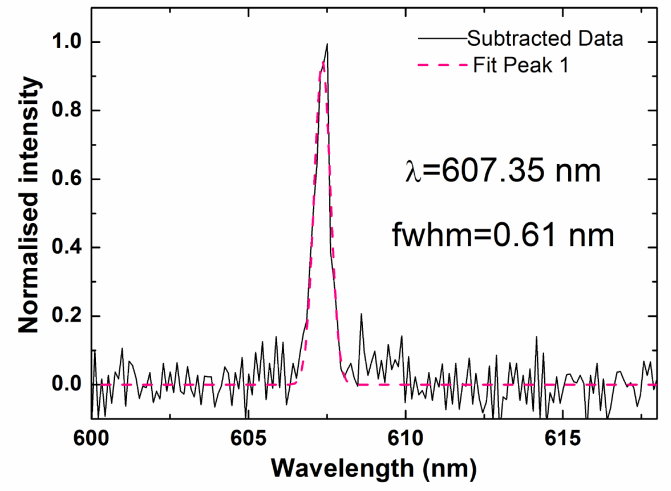

(b)

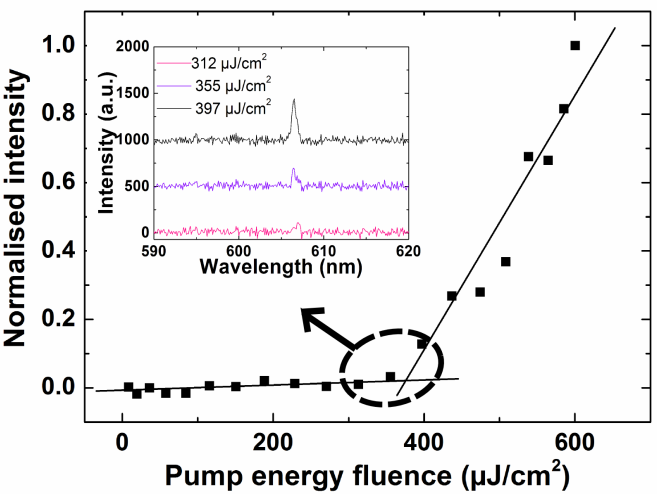

Figure 3: (a) and (b) represent respectively the spectrum and the power transfer function of the CQDs DFB laser on a flexible glass substrate. Inset of (b) gives details of the spectrum around the threshold region.

The behaviour of the hybrid CQD DFB lasers when bending the flexible glass substrate was studied. The emission wavelength above threshold for different bending radii of curvature is shown in figure 4. For these measurements, the pump energy was kept constant at $1.80 \mu \mathrm{J}$. Reducing the radii of curvature to -0.7 and $-0.5 \mathrm{~cm}$ induces a blue shift of the wavelength by $5 \mathrm{~nm}$ and $8 \mathrm{~nm}$, respectively. For radii of curvature of $0.6 \mathrm{~cm}$ and $0.4 \mathrm{~cm}$, the wavelength red-shifts by $6 \mathrm{~nm}$ and $10 \mathrm{~nm}$, respectively. The total tuning of $18 \mathrm{~nm}$ is limited by a maximum radius of curvature before fracture of the device and not limited by the gain bandwidth of the CQDs. 


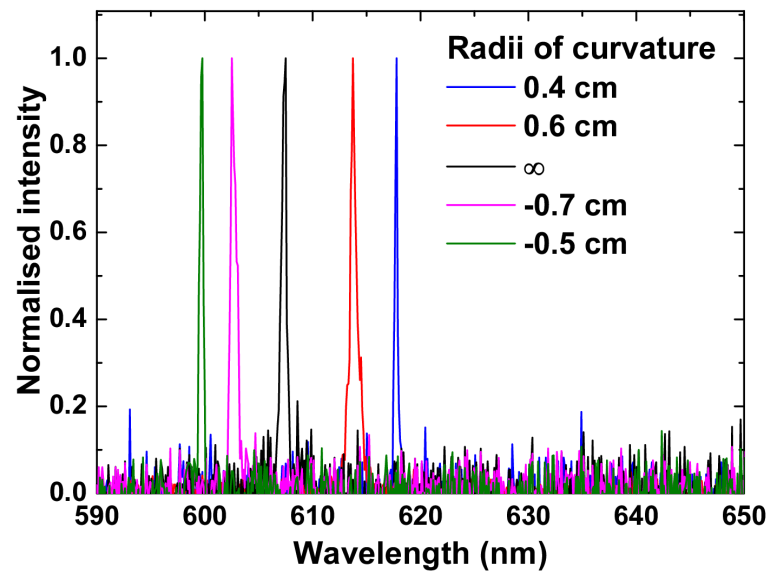

Figure 4: Shift in the emission wavelength of the flexible hybrid DFB laser on glass

It was verified that the emission wavelength does not change significantly as a function of the pump fluence, providing assurance that the tuning comes from the bending of the device. These shifts of the emission wavelength are attributed to corresponding changes in the period of the grating as the glass substrate is bent. The lasing mechanism when bending the sample can simply be explained by the Bragg equation. From equation 1 , it is clearly seen that a change in grating period will engender a change in wavelength. For a constant effective refractive index, a bigger period will increase the wavelength and a smaller period will reduce the wavelength. The change in period can be approximated from these wavelength measurements by using Eq. 1 and assuming that the effective refractive index is constant for the considered bending radii. Knowing that for a period of $390 \mathrm{~nm}$ (flat substrate) the emission wavelength is $607.35 \mathrm{~nm}$, the effective refractive index for the guided mode can be deduced ( 1.558). Then, for all the other emission wavelengths obtained when bending the laser, the corresponding 'altered' period, $\Lambda^{\prime}$, is calculated. The corresponding radius of curvature of the laser is determined geometrically using eq. (2). It is assumed here that the thickness of the gain material stays constant upon bending. Knowing the total thickness of the laser (CQD layer, epoxy grating and glass membrane), the period of the grating with $\left(\Lambda^{\prime}\right)$ and without $(\Lambda)$ bending, the radius of curvature is given by:

$$
\Lambda^{\prime}=\frac{\mathrm{R}+\mathrm{h}}{\mathrm{R}} \Lambda \Rightarrow \mathrm{R}=\frac{\mathrm{h} \Lambda}{\Lambda^{\prime}-\Lambda}
$$

In Eq. $2, \mathrm{R}$ is the radius of curvature, $\mathrm{h}$ the thickness of the laser structure, $\Lambda$ the initial period of the grating and $\Lambda^{\prime}$ is the period of the grating under bending. The values of the grating period and radius of curvature thus calculated are plotted as a function of the wavelength in Figure 5 (open triangles).

Theoretical values for the radii of curvature and the grating periods can be calculated and then compared to the values deduced previously from the experimental data. From buckling theory and Euler's column formula, the radius of curvature can be calculated as follow: ${ }^{18}$

$$
R= \pm e\left[1-\cos \sqrt{\left.\frac{P}{E I} x\right]}\right.
$$

In Eq. (3), e is the distance separating the sample from the neutral axis, $P$ is the allowable load, $E$ is the modulus of elasticity, $\mathrm{I}$ is the moment of inertia and $\mathrm{x}$ is the horizontal displacement applied on the micrometre screw of the mechanical stage (see Figure 2). The sign of the equation changes according to a negative or positive bending (see Figure 2). Euler's equation states that: ${ }^{19}$ 


$$
\mathrm{P}=\frac{\pi^{2} \mathrm{EIn}}{\mathrm{L}^{2}}
$$

In Eq. (4) $L$ is the initial length of the sample and $n$ is a coefficient for end conditions. In our case, $\mathrm{n}=0.5$ as the sample behaves as a fixed-fixed column ${ }^{20,21}$. Therefore Eq. (4) becomes:

$$
\frac{\mathrm{P}}{\mathrm{EI}}=\frac{0.5 \pi^{2}}{\mathrm{~L}^{2}}
$$

Using Eq. (3) and (5) leads to:

$$
\mathrm{R}= \pm \mathrm{e}\left[1-\cos \left(\frac{\pi}{\mathrm{L}} \sqrt{0.5 \mathrm{x}}\right]\right)
$$

Knowing the displacement applied from the micrometre screw, the radius of curvature is calculated for each measurement using Eq. 6. Finally, using those values of radii of curvature, the grating period is deduced with Eq. 2 as previously. These theoretical results are plotted (open squares) as a function of the emission wavelength in Figure 5 for comparison with the values obtained directly from the experimental data, showing good agreement. In Figure 6, the evolution of the wavelength as a function of the horizontal displacement $\mathrm{x}$ is displayed. The ascending part of the plot represents the red shift of the wavelength (positive curvature of the laser) and the descending line represents the blue shift or negative curvature, both having a slope of $\pm 19 \mathrm{~nm} / \mathrm{cm}$.

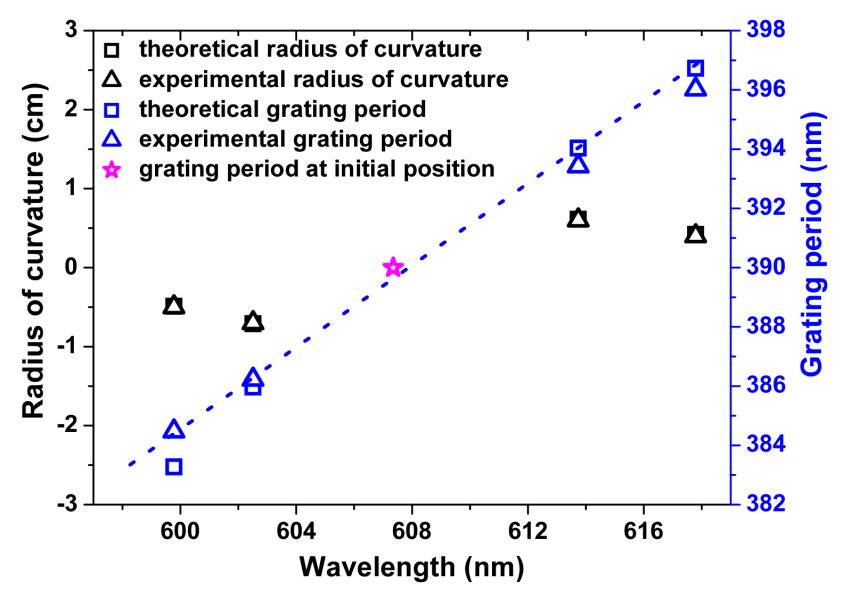

Figure 5: Comparison of theoretical and experimental values of the grating period and radius of curvature for each emission wavelength obtained after bending the CQD laser

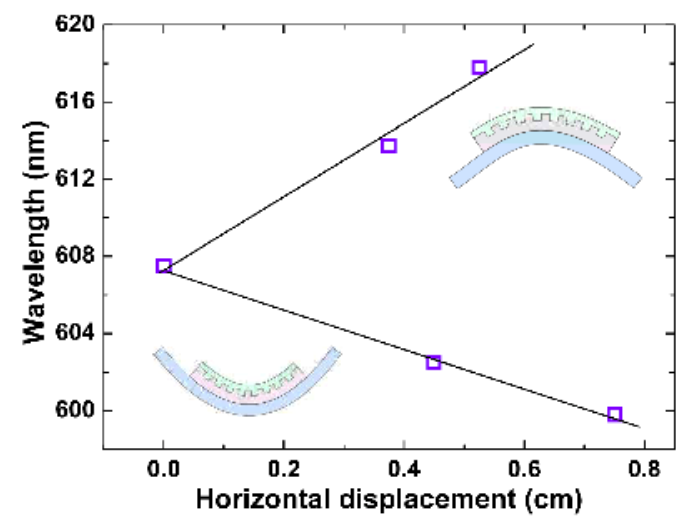

Figure 6: Evolution of the emission wavelength of the laser as a function of the horizontal displacement of the micrometre. Lines: theory; Open squares: data points. 
The lasing threshold being related to the grating period, a bigger or a smaller period would affect the value of the threshold. To determine the influence of the bending on the lasing threshold, the laser mode transverse intensity profile and its overlap with the gain region were calculated. This overlap, characterised by the overlap factor as defined further, is important because it influences the modal gain and hence the laser threshold (a higher overlap factor leading to a lower threshold in principle). The calculations were made by considering slab waveguides equivalent to the laser structures, i.e. with the appropriate refractive indices and thicknesses, and using a multilayer matrix model in order to determine the TEO intensity profile across the whole laser structure. In the case of our laser the layers to be considered are the gain material (the CQDs) enclosed between a semi-infinite epoxy substrate (NOA85) and air. The effective index for the TEO mode was first determined and the mode intensity profile subsequently derived. The overlap factor was deduced from the profile by the relation $\frac{\int_{\text {active_region }} \mathrm{I}(\mathrm{z}) \cdot \mathrm{dz}}{\int_{-\infty}^{+\infty} \mathrm{I}(\mathrm{z}) \cdot \mathrm{dz}}$ where the integral in the numerator is taken over the thickness of the gain region (see Figure 1), $z$ is the direction of the stack of layers, i.e. the transverse direction of the laser structure, and $\mathrm{I}(\mathrm{z})$ is the mode intensity profile. ${ }^{11}$ The calculated overlaps showed slightly varied when the period was changed. The threshold would be increased when the period is increased and improved when the period is decreased. However these changes are extremely small and probably negligible if we consider the possible defects in the laser structure engendering threshold variations (see figure $\mathrm{S} 2$ ).

In summary, a CQD laser on an ultra-thin glass membrane was demonstrated. The device is a mechanically-flexible, second-order DFB laser that combines colloidal quantum dots, a surfacepatterned polymeric film and an ultra-thin glass substrate. It operates in a single transverse mode, with an average threshold fluence of $450 \pm 80 \mu \mathrm{J} / \mathrm{cm}^{2}\left(90 \pm 16 \mathrm{~kW} / \mathrm{cm}^{2}\right)$ for an emission close to $607.5 \mathrm{~nm}$. The emission wavelength of the device is continuously-tunable over $18 \mathrm{~nm}$ by mechanically flexing the thin-glass substrate. The confirmed correlation between the curvature of the glass substrate and the emission wavelength will serve as a guideline for the future design of such tunable lasers. These lasers could find applications in areas including (bio-) sensing thanks to the nature of their substrate and their overall mechanical and optical properties.

\section{Acknowledgments}

This work was supported by EPSRC grants EP/J021962/1 and EP//029141/1. 


\section{References}

${ }^{1}$ Klimov, V.I., Los Alamos Science, 28, 214 (2003).

${ }^{2}$ Kim, T.-H., Cho K.-S., Lee, E.K., Lee, S.J., Chae, J., Kim, J.W., Kim, D.H., Kwon, J.-Y., Amaratunga, G., Lee, S.Y., Choi, B.L., Kuk, Y., Kim, J.M. and Kim, K., Nature Photonics, 5, 182 (2011).

${ }^{3}$ Sun, L., Choi, J.J, Stachnik, D. , Bartnik, A.C., Malliaras, G.G, Hanrath, T. and Frank W. Wise, F.W., Nature Photonics, 7, 369 (2012).

${ }^{4}$ Hoogland, S., V. Sukhovatkin, V., I. Howard, I., S. Cauchi, S., L. Levina, L., and E. H. Sargent, E.H., Optics Express, 14, 3273 (2006).

${ }^{5} \mathrm{Li}$, J.and Zhu, J.-J, Analyst, 138, 2506 (2013).

${ }^{6}$ Segev-Bar, M.H.and Haick, H., ACS Nano, 7, 8366 (2013).

${ }^{7}$ Kupfmüller, J., Stadlmann, K., Li, Z., Satzinger, V., Stampfl, J. and Liska, R., MRS Online Proceedings Library, 1438, 1438 (2013).

${ }^{8}$ Singh, R.S., Culjat, M.O., Vampola, S.P., Williams K., Grundfest, W.S., Lee, H. and Brown, E.R., IEEE Ultrasonics Symposium, 1824 (2007).

${ }^{9} \mathrm{Hu}$, J., Li, L., Lin, H., Zhang, P., Zhou, W., and Ma, Z.,. Optics Materials Express,. 3, 1313 (2013).

${ }^{10}$ http://www.us.schott.com.

${ }^{11}$ Foucher, C., Guilhabert, B., Kanibolotsky, A.L., Skabara, P.J., Laurand, N.and M.D. Dawson, M.D., Optics Materials Express, 2013. 3, 584.

${ }^{12}$ Lo, P.-Y., Ding, J.-M., Hu, J.-P., Chan, Y.-J., Garner, S., He, M., Lin, J., Li, X., Sorensen, M., Li, J.,Cimo, P. and Kuo, C., SID Symposium Digest of Technical Papers, 42, 387 (2011).

${ }^{13}$ Hoehla, S., Garner, S., Hohmann, M., Kuhls, O., Li, X., Schindler, A. and Fruehauf, N., Journal of Display Technology, 8, 309 (2012).

${ }^{14}$ Wu, K.-W., Wu, K.-W., Liao, Y.C., Shiu, J.W., Tsai, Y.S., Chen, K.T., Lai, Y.C., Lai, C.-C., Lee, Y.Z., SID Symposium Digest of Technical Papers, 42, 389 (2011).

${ }^{15}$ Sun, D., Miyatake, N. and Sue, H.-J., Nanotechnology, 18, (2007).

${ }^{16}$ Herrnsdorf, J., Guilhabert, B., Chen,Y., Kanibolotsky,A.L., Mackintosh, A.R., Pethrick, R.A., Skabara, P.J., Gu, E., Laurand, N.and M.D. Dawson, Optics Express, 18, 25535 (2010).

${ }^{7}$ Dang, C., Lee, J., Breen, C., Steckel, J.S, Coe-Sullivan, S. and Nurmikko, A., Nature Nanotechnology,. 7, 335 (2012).

${ }^{18}$ Higdon, A., Ohlsen, E.H. and Stiles, W.B., Mechanics of materials, London : Wiley \& Sons, 510 (1960).

${ }^{19}$ Gere, J.M.and Goodno, B.J., Mechanics of materials., SI Edition, 914 (2013).

${ }^{20}$ Beer, F.P., Johnston Jr, E.R., Dewolf, J.T. and Mazurek, D., Mechanics of Materials., Boston, Mass. : McGraw-Hill Higher Education, 617 (2009).

${ }^{21}$ Mott, R.L., Statics and strength of materials., Boston: Prentice Hall, 594 (2010).

${ }^{22}$ http://www.norlandprod.com/. 